\title{
Kitasato Symposium 2011: Translational prospects for cytokines in 2011
}

\author{
Potsdam, Germany. 22-23 September 2011 \\ Edited by Gerd R Burmester, Peter E Lipsky and Thomas Dörner \\ Published: 16 September 2011 \\ These abstracts are available online at http://arthritis-research.com/supplements/13/S2
}

\section{INTRODUCTION}

11

Kitasato symposium 2011: translational prospects for cytokines

Gerd R Burmester', Peter E Lipsky², Thomas Dörner ${ }^{2^{*}}$,

the Kitasato Meeting Faculty

'Dept. Medicine/Rheumatology and Clinical Immunology Charite University Medicine, Berlin, Germany; ${ }^{2}$ Charlottesville, NC, USA

Arthritis Research \& Therapy 2011, 13(Suppl 2):11

After successful meetings in 2009 and 2010, an international faculty of largely immunologists and rheumatologists will gather in Potsdam on September $22^{\text {nd }}$ and $23^{\text {rd }}, 2011$ to discuss the impact of cytokines in health and their contributions to autoimmunity in a symposium named after Shibasaburo Kitasato (1853 - 1931), who worked in Berlin between 1885 and 1892. During this rather short time, he together with Emil von Behring discovered the causative pathogens of tetanus and diphtheria and contributed substantially to our basic understanding of the interaction of the immune system and invading pathogenic microorganisms In keeping with the tradition of Kitasato, a major theme of the symposium will be the translation of basic science principles into understanding human disease. The keynote lecture of the 2011 Kitasato symposium will be delivered by Antonio Lanzevecchia (Belinzona/Switzerland) who has contributed many novel insights into understanding of immune regulation and host defense. His lecture is entitled "Dissecting the human immune response to pathogens".

This year's Kitasato Symposium will be a joint meeting with the Research Collaborative Consortium (Sonderforschungsbereich) 650 "Cellular approaches to the suppression of unwanted immune reactions - from bench to bedside". As in previous years, the Kitasato symposium will focus on mechanisms of autoimmunity and tolerance emphasizing the role of cytokines. A deeper understanding of these aspects and adapyive and innate immunity has paved the way to innovative therapies for autoimmune disease within the last decade, especially in rheumatoid arthritis (inhibition of TNF, IL-1 and IL-6R) and very recently in systemic lupus erythematosus (BAFF/BLyS blockade).

In specific sessions, the role of tolerance in autoimmunity as well as transplantation, signaling pathways in cytokine stimulation, the analysis of new cytokine targets, and the translational of cytokine biology into human disease will be discussed. The Symposium will especially focus on novel developments within the last few years with the promise of yielding new targets for therapy. In addition, insights on disease biology developing from the clinical use of biologis will be highlighted.

It is the promise of the meeting to provide new perspectives of basic, translational and clinical research in the field serving the ultimate goal of improving the treatment of patients. The collection of the individual contributions is summarized in the following abstract supplement.

\section{KEYNOTE LECTURE PRESENTATION}

\section{K1}

Dissecting the human $\mathrm{T}$ and $\mathrm{B}$ cell response to pathogens

Antonio Lanzavecchia ${ }^{1,2}$

${ }^{1}$ Institute for Research in Biomedicine, Bellinzona, Switzerland; ${ }^{2}$ Institute of

Microbiology ETH-Zurich, Zurich, Switzerland

Arthritis Research \& Therapy 2011, 13(Suppl 2):K1

Memory $\mathrm{T}$ and $\mathrm{B}$ lymphocytes and long lived plasma cells represent a repository of the antigenic experience of an individual. By analyzing the specificity and function of these cells we can gain insights into the human immune response and identify mechanisms of protection and immunopathology. We have developed methods to dissect the functional heterogeneity and antigenic repertoire of human T cells, B cells and plasma cells. These methods are used: i) to identify subsets of effector and memory $T$ cells with distinct roles in immune surveillance and protection in different tissues against different classes of pathogens, and ii) to dissect the relative role of plasma cells and memory $B$ cells in the antibody response to pathogens and to isolate broadly neutralizing antibodies. A better understanding of the class and specificity of the human immune response will be instrumental to guide the design of effective vaccines.

\section{ORAL PRESENTATIONS}

01

Targeting activated T cells in type I diabetes and islet transplantation Lucienne Chatenoud

Paris, France

Arthritis Research \& Therapy 2011, 13(Suppl 2):O1

Abstract not submitted at time of publication

02

Inducing a tolerogenic microenvironment within tissues Stephen P Cobbold

Sir William Dunn School of Pathology, University of Oxford, Oxford, OX1 3RE, UK E-mail: stephen.cobbold@path.ox.ac.uk

Arthritis Research \& Therapy 2011, 13(Suppl 2):O2

The maintenance of tolerance to both self tissues foreign organ grafts depends on the activity of foxp $3^{+}$regulatory $T$ cells (Treg). We have used MHC-matched skin grafts as a model system to study how such Treg can be induced therapeutically and the mechanisms by which they act. Using 
monospecific TCR transgenic mice we have shown that a short treatment with monoclonal antibodies that block full T cell activation in vivo allows the targeted tissue to itself induce de novo, antigen specific, foxp $3^{+}$Treg (iTreg) [1]. We also show that these iTreg are not only concentrated within the target tissue, but are continuously required to suppress the activity of primed effector cells also present within the tissue [2]. When taken together with previous findings of linked suppression and infectious tolerance [3], the evidence suggests that tolerance maintained by iTreg is dependent on a local, tolerogenic microenviroment within the tissue. One component of this microenvironment is the induction, by both innate inflammation and iTreg, of multiple enzymes that consume essential amino acids, including tryptophan, arginine and valine. Local amino acid depletion can be sensed by naïve and effector T cells, via the mammalian target of the immunosuppressive drug rapamycin (mTOR) pathway, which can synergise with TGF $\beta$ for the further induction of foxp3 ${ }^{+}$iTreg [4]. TGF $\beta$ is also able to up-regulate the ectoenzymes CD39 and CD73 both on T cells and antigen presenting cells to catabolise inflammatory ATP to anti-inflammatory adenosine [5]. Microarray analysis of tolerated and control skin grafts for patterns of gene expression associated with the tolerogenic microenvironment confirms that these mechanisms are preferentially active locally within the tolerated tissues rather than throughout the systemic lymphoid system. Of particular interest, these same mechanisms seem to be active in grafted syngeneic tissues [6], suggesting that iTreg maintained microenvironments are important for maintaining self tolerance in the face of an inflammatory insult. The challenge now is how we can exploit appropriate combinations of T cell blockade, mTOR inhibition and TGF $\beta$ activation for translation to the clinic.

References

1. Cobbold SP, Castejon R, Adams E, Zelenika D, Graca L, Humm S, Waldmann $\mathrm{H}$ : Induction of foxp3+ regulatory $\mathrm{T}$ cells in the periphery of $\mathrm{T}$ cell receptor transgenic mice tolerized to transplants. J Immunol 2004, 172:6003-6010

2. Kendal AR, Chen Y, Regateiro F, Ma J, Adams E, Cobbold SP, Hori S, Waldmann H: Sustained vigilance of Foxp3+ T cells is vital for infectious tolerance in vivo. $J$ Exp Med 2011 in press.

3. Cobbold SP, Adams E, Nolan KF, Regateiro FS, Waldmann H: Connecting the mechanisms of $T$ cell regulation: dendritic cells as the missing link. Immunological Reviews 2010, 236:203-218.

4. Cobbold SP, Adams E, Farquhar CA, Nolan KF, Howie D, Lui KO, Fairchild PJ, Mellor AL, Ron D, Waldmann H: Infectious tolerance via the consumption of essential amino acids and mTOR signaling. Proc Natl Acad Sci USA 2009, 106(29):12055-60

5. Regateiro FS, Howie D, Agorogiannis El, Greaves DR, Cobbold SP, Waldmann H: CD73 expression and adenosine generation are controlled by TGF- $\beta$ and modulated by inflammatory cytokines. Eur J Immunol 2011 in press.

6. Cobbold SP, Adams E, Waldmann H: Biomarkers of transplantation tolerance: more hopeful than helpful? Front Immun 2011, 2:9, p. 1-8. doi:10.3389/fimmu.2011.00009.

03

Peptides reloaded - new strategies for tolerogenic vaccination Alf Hamann ${ }^{* *}$, Jennifer Pfeil ${ }^{1}$, Elisabeth Kenngott ${ }^{1}$, Tony Pernthaner ${ }^{2}$ Susanne Hartmann ${ }^{3}$, Ute Hoffmann

'Experimental Rheumatology, Charite Universitätsmedizin and Deutsches

Rheuma-Forschungszentrum Berlin, Germany; ${ }^{2}$ AgResearch, Hopkirk Research Institute, Massey University, Palmerston North, New Zealand; ${ }^{3}$ Inst.

Parasitology, Humboldt University Berlin, Germany

Arthritis Research \& Therapy 2011, 13(Suppl 2):O3

Uncontrolled immune reactions, e.g. in autoimmunity, chronic inflammation or allergy are a major cause of chronic and partially life-threatening diseases. Current treatments including those involving biologics largely rely on unspecific suppression of the effector cells and rarely are able to cure the disease. The native mechanisms of tolerance, notably those of active suppression by regulatory cells, have therefore fascinated immunologists from the beginning on as they promise modulation of the immune system in an antigen-specific way. However, early attempts to achieve tolerance by oral immunization or peptide vaccination worked in mouse models, but hardly were successful in humans. This might have two reasons: a), the modes of tolerogenic vaccination might not be very efficient, and $b$ ), the abundance of inflammatory effector/memory cells in adult humans might prevent induction and functioning of regulatory cells.

Our group is presently focusing on the first point and designing novel modifications of peptide-based vaccines able to induce regulatory cells. Conjugation of peptides to carrier molecules is one way to improve their in vivo efficacy in inducing Foxp3+ Tregs. In an EAE model, an improved protective efficacy can be demonstrated. A second approach is aiming to target the antigen to the gastrointestinal route which is usually associated with tolerization rather than effector response. Use of signal molecules targeting the peptides to epithelial transport mechanisms is presently explored to improve the efficacy of intestinal vaccination. Finally, we investigate immunomodulatory substances produced by parasites for a potential use as tolerogenic adjuvants. While these approaches might help to improve feasibility of peptide vaccination to induce tolerance, we assume that treatment of existing autoimmune disease will require a combination therapy which incorporates elimination of inflammatory effector cells or suppression of their activity.

\section{4}

Regulatory T cells in transplantation - from preclinical models to clinical study

Kathryn Wood

Transplantation Research Immunology Group, Nuffield Department of Surgical Sciences, University of Oxford, John Radcliffe Hospital, Oxford OX3

9DU, UK

Arthritis Research \& Therapy 2011, 13(Suppl 2):O4

After exposure to alloantigen in vivo and in vitro, alloantigen reactive immunoregulatory activity is enriched in a population of $\mathrm{CD}^{+} \mathrm{T}$ cells that express high levels of $C D 25$, the $\alpha$ chain of the interleukin-2 receptor and the transcription factor FOXP3. In vivo, common mechanisms underpin the activity of $\mathrm{CD} 25^{+} \mathrm{CD} 4^{+}$Treg in adult hosts. We identified a unique role for IFN $\gamma$ in the functional activity of $\mathrm{CD}_{2} 5^{+} \mathrm{CD} 4^{+}$alloantigen reactive Treg during the development of operational tolerance to donor alloantigens in vivo that is consistent with observations showing that tolerance to alloantigens cannot be induced in the absence of IFN $\gamma$ [1]. In order to provide proof of concept data for translation of findings in preclinical models to the bedside, we have demonstrated that human regulatory $T$ cells expanded ex vivo can protect human allografts (skin and vessels) from rejection [2,3].

The identification and characterisation of Treg that can control immune responsiveness to alloantigens has opened up exciting opportunities for new therapies in transplantation.

\section{References}

1. Sawitzki B, Kingsley Cl, Oliveira V, Karim M, Herber M, Wood KJ: Interferon gamma production by alloantigen reactive $\mathrm{CD}_{2} 5^{+} \mathrm{CD} 4^{+}$regulatory $\mathrm{T}$ cells is important for their regulatory function in vivo. Journal of Experimental Medicine 2005, 201:1925-1935.

2. Nadig SN, Wieckiewicz J, Wu DC, Warnecke G, Zhang W, Luo S, et al: In vivo prevention of transplant arteriosclerosis by ex vivo-expanded human regulatory T cells. Nat Med 2010, 16(7):809-813.

3. Issa F, Hester J, Goto R, Nadig SN, Goodacre T, Wood K: Ex vivo-expanded human regulatory $T$ cells prevent the rejection of skin allografts in a humanised mouse model. Transplantation 2010, 90:1321-1327.

05

Relevance and targeting of memory $\mathrm{T}$ cells in transplantation Birgit Sawitzki ${ }^{1,2^{*}}$, Anja Siepert ${ }^{3}$, Manfred Lehmann ${ }^{3}$, Undine Gerlach ${ }^{4}$ Andreas Pascher ${ }^{4}$, Stefan Tomiuk, ${ }^{5}$, Hans-Dieter Volk ${ }^{1,2}$, Petra Reinke ${ }^{2,6}$ ${ }^{1}$ Institute of Medical Immunology, Charite Universitätsmedizin, Berlin, Germany; ${ }^{2}$ Berlin Brandenburg Center for Regenerative Medicine, Berlin, Germany; Institute of Medical Biochemistry and Molecular Biology, University of Rostock, Rostock, Germany; ${ }^{4}$ Department of Visceral and Transplant Surgery, Charite Universitätsmedizin, Berlin, Germany; ${ }^{5}$ Miltenyi Biotec GmbH, Bergisch-Gladbach, Germany; ${ }^{6}$ Department of Nephrology and Internal Intensive Care Medicine, Charite Universitätsmedizin, Berlin, Germany Arthritis Research \& Therapy 2011, 13(Suppl 2):O5

Achieving tolerance or drug minimization after transplantation and thus preventing permanent immunosuppression with all the known severe 
side effects is the most important goal in transplantation medicine. In the last 20 years major progress has been made in understanding the tolerance underlying mechanisms and develop therapeutic strategies in small animal models. However, such knowledge could be rarely translated into the development of successful new therapeutic approaches in clinical transplantation. The success is limited by clinical challenges which are not present in our clean animal facilities such as 1) heterologous immunity - pathogen-specific memory $T$ and $B$ cells recognize alloantigens and boost the immune response towards the allograft, 2) pre-sensitization of recipients - presence of allo-specific memory $T$ and $B$ cells which are inert to most known therapeutic regimens. Thus we know now that we need more personalized treatment strategies according to the patient's immune reactivity. Such a strategy should combine three important aspects: i) an improved immune monitoring; ii) treatment which target memory cells and iii) strategies to reinforce regulation

We have established preclinical transplant models with preformed alloreactive or pathogen-specific memory $T$ cells in which we compare effectiveness of different treatment approaches combining depletional with regulatory approaches.

Furthermore, we have performed a DNA microarray screen on samples of transplant patients and identified surface molecules specifically expressed by naïve, central memory, effector memory or terminal differentiated effector memory (TEMRA) T cells. Using this approach we hope to develop antibodies, which specifically deplete effector memory and TEMRA cells but spare naïve and central memory T cells. Such a treatment will be associated with less side effects e.g. infectious complications as compared to global depletion of $\mathrm{T}$ and $\mathrm{B}$ cells.

\section{6}

Tregs combined with mature donor $\mathrm{T}$ cells hasten immune reconstitution without triggering GvHD in HLA haploidentical transplantation

Mauro Di lanni ${ }^{12^{*}}$, Franca Falzetti', Massimo F Martelli ${ }^{1}$

${ }^{1}$ Hematology and Clinical Immunology Section, Department of Clinical and Experimental Medicine, University of Perugia, Italy; ${ }^{2}$ Weizmann Institute of Science, Immunology Department, Rehovot, Israel

Arthritis Research \& Therapy 2011, 13(Suppl 2):O6

Haploidentical transplantation, with extensive $\mathrm{T}$ cell depletion to prevent GvHD, is associated with a high incidence of infection-related deaths. The key challenge is to improve immune recovery with allogeneic donor T cells without triggering GvHD. As T regulatory cells (Tregs) controlled GvHD in preclinical studies, the present phase I/II clinical trial evaluated the impact of early infusion of donor CD4/CD25+ Tregs, followed by an inoculum of donor mature T cells (Tcons) and positively immunoselected CD34+ cells. Twentyeight patients (median age 41, range 21-60) were enrolled from September 2008 onwards; 22 had AML (10 in CR1 at high risk, 10 in $\geq C R 2$ and 2 in relapse), 5 had ALL (4 in CR1; 1 in relapse) and 1 had high grade NHL in relapse. Conditioning was: 8 Gy single fraction TBI, thiotepa $(4 \mathrm{mg} / \mathrm{kg} \times 2)$, fludarabine $\left(40 \mathrm{mg} / \mathrm{m}^{2} \times 5\right)$, cyclophosphamide $(35 \mathrm{mg} / \mathrm{kg} \times 2)$. All patients received immunoselected Tregs (CliniMACS, Miltenyi Biotec) $(23 / 282 \times$ $10^{6} / \mathrm{kg} \mathrm{bw} ; 5 / 284 \times 10^{6} / \mathrm{kg}$ bw) and 4 days later positively immunoselected CD34+ cells (median $8.2 \times 10^{6} / \mathrm{kg} \mathrm{bw}$, range 5.0-19.1) together with Tcons $\left(4 / 280.5 \times 10^{6} / \mathrm{kg}\right.$ bw; $17 / 281 \times 10^{6} / \mathrm{kg}$ bw; $5 / 282 \times 10^{6} / \mathrm{kg} \mathrm{bw} ; 2 / 28 \mathrm{did}$ not receive Tcons). CD4/CD25 $5^{+}$Tregs (purity $92.7 \pm 2.1$ ) consisted of $33.6 \% \pm$ $13.1 \mathrm{CD} 25^{\text {high }} ; 58.1 \% \pm 6.6 \mathrm{CD} 25^{\text {int }} ; 5.8 \% \pm 2.5 \mathrm{CD} 25^{\text {low }} ; 65.7 \% \pm 11.8$ FoxP3; $17.4 \% \pm 7.2$ CD127 (mean \pm SD). No GvHD prophylaxis was administered. 26/28 patients engrafted. No GvHD developed in 24/26 patients, 2 developed $\geq$ grade II GvHD. Ten patients died (3 VOD, 2 fungal pneumonia, 1 bacterial sepsis, 1 CNS aspergillosis, 1 systemic toxoplasmosis, 1 adenoviral infection, 1 MOF). CD4 and CD8 counts reached, respectively, 50/ $\mu \mathrm{L}$ medianly on days 34 (range 19-63 days) and 24 (range 15-87); 100/ $\mu$ L medianly on days 47 (range 28-100 days) and 34 (range 19-95); 200/ $\mathrm{LL}$ on days 70 (range 41-146 days) and 61 (range 21-95). A wide T-cell repertoire developed rapidly with high frequencies of specific CD4+ and CD8+ for opportunistic pathogens. Episodes of CMV reactivation were significantly fewer than after our "standard haplo" transplants. In KIR ligand-mismatched transplants, speed of NK cell reconstitution/maturation and size of donor vs recipient alloreactive NK cell repertoires were preserved. In conclusion, in the setting of haploidentical transplantation infusion of Tregs makes administration of a high dose of T cells feasible for the first time. This strategy provides a longterm protection from GvHD and robust immune reconstitution.

07

Natural Treg and role of IL-2 in lupus

Gabriela Riemekasten*, Jens Y Humrich

Department of Rheumatology and Clinical Immunology, Charité University

hospital, Berlin, 10117, Germany

Arthritis Research \& Therapy 2011, 13(Suppl 2):O7

Background: Effector T cells play an important role in the pathogenesis of lupus. As recently shown in murine lupus, they contribute to tissue damage and glomerulonephritis.

Methods: The role of naturally occurring regulatory T cells (Treg) and of IL-2 was studied in vitro and in vivo by using flow cytometry and the NZB/W lupus mouse model.

Results: In healthy individuals as well as in young lupus prone mice without any signs of the disease, effector T cells are tightly controlled by naturally occurring regulatory T cells (Treg) that can be shown by different approaches: 1. After depletion of Treg cells by anti-CD25 therapy, murine lupus is strongly accelerated. 2. After passive transfer of Treg (CD4+CD25+ T cells consisting of 95\% FoxP3+ T cells), murine lupus improved reflected by reduced proteinuria and increased survival compared to control mice [1]. 3. In vitro depletion of Treg lead to better detection of autoantigen-specific effector T cells with frequencies above the detection limit for flow cytometry. The frequency of autoangien-specific $T$ cells correlate with the disease activity in human and murine lupus. The control of effector T cells by Treg cells can be also used for studying the phenotype of effector $\mathrm{T}$ cell and their function at an autoantigen-specific level. However; as shown in the murine NZB/W lupus model, there is a progressive loss of Treg/Tcon homeostasis during lupus development in different compartments with a progressive Treg deficiency. In lupus mice with proteinuria, the phenotype of effector $T$ cells is very similar to the T cell phenotype obtained in IL-2 deficient mice. As known from the literature, IL-2 levels are decreased in SLE patients. According to the characteristics of Treg, they are more sensitive to IL-2 deficiency. Supporting this, addition of IL-2 resulted in a dominant proliferation of Treg cells. In murine lupus, IL-2 improved survival and decreased proteinuria in diseased NZB/W mice.

Conclusions: Our data support the possible role of Treg and of IL-2 supplementation in lupus therapy. Further studies are underway to evaluate IL-2 supplementation and its effects on immune cells and disease symptoms in lupus.

Reference

1. Humrich JY, Morbach $H$, Undeutsch $R$, Enghard $P$, Rosenberger $S$,

Weigert O, Kloke L, Heimann J, Gaber T, Brandenburg S, Scheffold A, Huehn J, Radbruch A, Burmester GR, Riemekasten G: Homeostatic imbalance of regulatory and effector T cells due to IL-2 deprivation amplifies murine lupus. Proc Natl Acad Sci USA 2010, 107(1):204-209.

08

Adipocytokines and autoimmunity

Klaus W Frommer, Elena Neumann, Ulf Müller-Ladner

Department of Internal Medicine and Rheumatology, Justus-Liebig University Gießen, Kerckhoff-Klinik, Bad Nauheim, D-61231, Germany

E-mail: u.mueller-ladner@kerckhoff.klinik.de

Arthritis Research \& Therapy 2011, 13(Suppl 2):08

By definition, adipo(cyto)kines are cytokine-like mediators produced mainly by adipose tissue. In the human body, they participate in regulating a variety of physiological functions related to energy metabolism [1] and inflammation [2]. Increased or decreased adipokine levels are associated with autoimmune diseases including diabetes mellitus type 1 (DMT1) [3] rheumatoid arthritis (RA) [4,5], ankylosing spondylitis (AS) [6], systemic sclerosis (SSc) [7], systemic lupus erythematosus (SLE) [8] and Behçet's disease [9-12].

In vitro data suggest that adipokines may contribute to the progression of RA as they are potent inducers of proinflammatory cytokines, chemokines and matrix metalloproteinases (MMP) in RA effector cells [13-16].

SSc also appears to be associated with adipokines. Contrary to RA, in which substantial intraarticular degradation of extracellular matrix occurs, SSC is 
characterized by excessive fibrosis. In vitro data and data from animal models of fibrosis point towards a dual role of the adipokine adiponectin as presented by numerous groups at the EULAR congress in London, specifically an antifibrotic effect in later stages of SSC and a profibrotic effect in earlier stages, which appears to be induced by proinflammatory cytokines. Likewise, leptin is involved in the development of liver fibrosis [17-19].

So far, no or only little functional information is available regarding the role of adipokines in other autoimmune diseases. Serum level and clinical correlation analyses, however, suggest an association with adipokines. In AS, elevated resistin serum levels have been found, while adiponectin levels remained unchanged $[6,20]$. On the other hand, leptin is discussed controversially in AS. Serum levels were decreased in AS according to two studies $[20,21]$, while they were increased according to another [22]. Also, while correlations of leptin with parameters of inflammation (C-reactive protein, IL-6) and disease activity (Bath Ankylosing Spondylitis Disease Activity Index) have been found by Park et al. [22], no such correlations could be found by Toussirout et al. [20]. Interestingly, peripheral blood mononuclear cells (PBMC) from AS patients express and secrete more leptin, IL- 6 and TNF- $\alpha$ than PBMC from control subjects. Additionally, stimulation of PBMC from AS patients with exogenous leptin led to a significantly increased IL- 6 and TNF- $\alpha$ production [23]. Hence, leptin might be involved in the pathogenesis of AS.

In SLE, resistin, for example, has been shown to be associated with general inflammation and bone loss, suggesting a proinflammatory and disease-promoting function [24].

However, the exact role of adipokines especially in SSc, AS and SLE is still unclear and will require further investigation. Also, further research is warranted to show whether adipokines may represent potential therapeutic targets in this diseases.

References

1. Rajala MW, Scherer PE: Minireview: The adipocyte-at the crossroads of energy homeostasis, inflammation, and atherosclerosis. Endocrinology 2003, 144:3765-3773.

2. Neumann E, Frommer KW, Vasile M, Muller-Ladner U: Adipocytokines as driving forces in rheumatoid arthritis and related inflammatory diseases? Arthritis Rheum 2011, 63:1159-1169.

3. Galler A, Gelbrich G, Kratzsch J, Noack N, Kapellen T, Kiess W: Elevated serum levels of adiponectin in children, adolescents and young adults with type 1 diabetes and the impact of age, gender, body mass index and metabolic control: a longitudinal study. Eur J Endocrinol 2007 157:481-489.

4. Schäffler A, Ehling A, Neumann E, Herfarth H, Tarner I, Schölmerich J, Müller-Ladner U, Gay S: Adipocytokines in synovial fluid. JAMA 2003, 290:1709-1710.

5. Laurberg TB, Frystyk J, Ellingsen T, Hansen IT, Jorgensen A, Tarp U, Hetland ML, Horslev-Petersen K, Hornung N, Poulsen JH, et al: Plasma adiponectin in patients with active, early, and chronic rheumatoid arthritis who are steroid- and disease-modifying antirheumatic drugnaive compared with patients with osteoarthritis and controls. J Rheumatol 2009, 36:1885-1891.

6. Kocabas H, Kocabas V, Buyukbas S, Melikoglu MA, Sezer I, Butun B: The serum levels of resistin in ankylosing spondylitis patients: a pilot study. Rheumatol Int 2010, [Epub].

7. Kotulska A, Kucharz EJ, Brzezinska-Wcislo L, Wadas U: A decreased serum leptin level in patients with systemic sclerosis. Clin Rheumatol 2001, 20:300-302.

8. Harle P, Pongratz G, Weidler C, Buttner R, Scholmerich J, Straub RH: Possible role of leptin in hypoandrogenicity in patients with systemic lupus erythematosus and rheumatoid arthritis. Ann Rheum Dis 2004 63:809-816.

9. Ozgen M, Koca SS, Dagli N, Balin M, Ustundag B, Isik A: Serum adiponectin and vaspin levels in rheumatoid arthritis. Arch Med Res 2010, 41:457-463.

10. Sezen H, Okumus S, Pehlivan Y, Dilli I, Tarakcioglu M, Onat AM: Visfatin Levels in Behcet's Disease. Inflammation 2011, [Epub]

11. Yalcindag FN, Yalcindag A, Batioglu F, Caglayan O, Kisa U, Ozdemir O: Evaluation of serum resistin levels in patients with ocular and nonocular Behcet's disease. Can J Ophthalmol 2008, 43:473-475.

12. Evereklioglu C, Inaloz HS, Kirtak N, Doganay S, Bulbul M, Ozerol E, Er H, Ozbek E: Serum leptin concentration is increased in patients with Behcet's syndrome and is correlated with disease activity. $\mathrm{Br} J$ Dermatol 2002, 147:331-336.
13. Ehling A, Schaffler A, Herfarth $H$, Tarner $\mathbb{H}$, Anders $\mathrm{S}$, Distler $\mathrm{O}$, Paul $\mathrm{G}$ Distler J, Gay S, Scholmerich J, et al: The potential of adiponectin in driving arthritis. J Immunol 2006, 176:4468-4478.

14. Frommer KW, Zimmermann B, Meier FM, Schroder D, Heil M, Schäffler A Büchler C, Steinmeyer J, Brentano F, Gay S, et al: Adiponectin-mediated changes in effector cells involved in the pathophysiology of rheumatoid arthritis. Arthritis Rheum 2010, 62:2886-2899.

15. Bokarewa M, Nagaev I, Dahlberg L, Smith U, Tarkowski A: Resistin, an adipokine with potent proinflammatory properties. J Immunol 2005, 174:5789-5795.

16. Brentano F, Schorr O, Ospelt C, Stanczyk J, Gay RE, Gay S, Kyburz D: Pre-B cell colony-enhancing factor/visfatin, a new marker of inflammation in rheumatoid arthritis with proinflammatory and matrix-degrading activities. Arthritis Rheum 2007, 56:2829-2839.

17. Ikejima K, Takei Y, Honda H, Hirose M, Yoshikawa M, Zhang YJ, Lang T, Fukuda T, Yamashina S, Kitamura T, Sato N: Leptin receptor-mediated signaling regulates hepatic fibrogenesis and remodeling of extracellular matrix in the rat. Gastroenterology 2002, 122:1399-1410.

18. Saxena NK, Ikeda K, Rockey DC, Friedman SL, Anania FA: Leptin in hepatic fibrosis: evidence for increased collagen production in stellate cells and lean littermates of ob/ob mice. Hepatology 2002, 35:762-771.

19. Wang J, Leclerca I, Brymora JM, Xu N, Ramezani-Moghadam M, London RM, Brigstock D, George J: Kupffer cells mediate leptin-induced liver fibrosis. Gastroenterology 2009, 137:713-723.

20. Toussirot E, Streit G, Nguyen NU, Dumoulin G, Le Huede G, Saas P, Wendling D: Adipose tissue, serum adipokines, and ghrelin in patients with ankylosing spondylitis. Metabolism 2007, 56:1383-1389.

21. Sari I, Demir T, Kozaci LD, Akar S, Kavak T, Birlik M, Onen F, Akkoc N: Body composition, insulin, and leptin levels in patients with ankylosing spondylitis. Clin Rheumatol 2007, 26:1427-1432.

22. Park MC, Lee SW, Choi ST, Park YB, Lee SK: Serum leptin levels correlate with interleukin-6 levels and disease activity in patients with ankylosing spondylitis. Scand J Rheumatol 2007, 36:101-106.

23. Park MC, Chung SJ, Park YB, Lee SK: Pro-inflammatory effect of leptin on peripheral blood mononuclear cells of patients with ankylosing spondylitis. Joint Bone Spine 2009, 76:170-175.

24. Almehed K, d'Elia HF, Bokarewa M, Carlsten H: Role of resistin as a marker of inflammation in systemic lupus erythematosus. Arthritis Res Ther 2008, 10:R15.

\section{9}

Orchestration of B and T cell responses in health and disease by common gamma chain family cytokines with a focus on IL-21 Manfred Kopf*', Luigi Tortola, Iwana Schmitz, Anja Fröhlich, Ivo Sonderegger, Helga Pawelski, Christoph Schneider

Institute of Integrative Biology, Molecular Biomedicine, ETH Zürich,

Switzerland

Arthritis Research \& Therapy 2011, 13(Suppl 2):09

Members of a subfamily of the type 1 four-helix-bundle cytokines with receptors sharing the common gamma $\left(c_{\gamma}\right)$ chain including IL-2, IL-4, IL-7, IL-9, IL-15, and IL-21 have distinct activities on the differentiation of effector, memory, and regulatory T cells [1,2]. Furthermore, IL-2, IL-4, and IL-21 serve distinct roles in control of $B$ cell development and differentiation to antibody producing cells. We and others recently reported that both IL-2 and IL-21 are essential for maintenance of CD8 T cells and control of chronic viral infection, while both cytokines are dispensable for expansion and contraction of CD8 T cells during acute and resolved viral infection [3-7]. While IL-21 has been implicated in cross-regulation of Th17 cells and inducible regulatory T cells (Treg) in vitro, development of Th17 and Treg cells and consequently organ-related autoimmune disease remain unaffected in IL-21R-deficient mice in vivo [8,9]. In contrast, we now found that IL-21 can potently inhibit proliferation and function of inducible and natural Treg cells in models of T cell transfer colitis, viral infection, and asthma. Increased numbers of Tregs in IL-21R-deficient mice offer an explanation for suppression of Th2-mediated asthma and susceptibility to chronic viral infection described in the knockout mice $[5,10]$.

Furthermore, the importance of IL-21 for B cell and antibody responses has been well established. Recently, it has been suggested that IL-21 is crucial for development of T follicular helper cells (Tfh) and defective B cell responses in IL-21R-deficient mice are due to the absence of Tfh cells. 
However, we found that germinal center development and antibody responses were severely impaired in mice that lack IL-21R specifically on B cells suggesting that IL-21 regulates germinal center responses in a $B$ cell intrinsic manner [11]. In addition, we have shown that requirement of IL-21 for a B cell response is overcome by immunization with particulate antigens containing TLR7/8 ligands (such as viral RNS). These data demonstrate that innate pathogen patterns (PAMPs) and Th cell derived signals co-operate in the induction of optimal IgG responses. Interestingly, in contrast to follicular B cell responses, IL-21 has been shown to negatively regulate marginal zone (MZ) B-cell survival and antibody production to Streptococous pneumonia [12].

References

1. Rochman $Y$, Spolski $R$, Leonard WJ: New insights into the regulation of T cells by gamma(c) family cytokines. Nat Rev Immunol 2009, 9:480-490,

2. Spolski R, Leonard WJ: Interleukin-21: basic biology and implications for cancer and autoimmunity. Annual review of immunology 2008, 26:57-79.

3. Bachmann MF, Wolint P, Walton S, Schwarz K, Oxenius A: Differential role of IL-2R signaling for CD8+ T cell responses in acute and chronic viral infections. European journal of immunology 2007, 37:1502-1512.

4. Elsaesser H, Sauer K, Brooks DG: IL-21 is required to control chronic viral infection. Science 2009, 324:1569-1572.

5. Fröhlich A, et al: IL-21R on T cells is critical for sustained functionality and control of chronic viral infection. Science 2009, 324:1576-1580

6. Williams MA, Tyznik AJ, Bevan MJ: Interleukin-2 signals during priming are required for secondary expansion of CD8+ memory T cells. Nature 2006 441:890-893.

7. Yi JS, Zajac AJ: A vital role for interleukin-21 in the control of a chronic viral infection. Science 2009, 324:1572-1576.

8. Coquet JM, Chakravarti S, Smyth MJ, Godfrey DI: Cutting edge: IL-21 is not essential for Th17 differentiation or experimental autoimmune encephalomyelitis. J Immunol 2008, 180:7097-7101.

9. Sonderegger I, Kisielow J, Meier R, King C, Kopf M: IL-21 and IL-21R are not required for development of Th17 cells and autoimmunity in vivo. European journal of immunology 2008, 38:1833-1838.

10. Fröhlich A, et al: IL-21 receptor signaling is integral to the development of Th2 effector responses in vivo. Blood 2007, 109:2023-2031.

11. Bessa J, Kopf M, Bachmann MF: Cutting Edge: IL-21 and TLR Signaling Regulate Germinal Center Responses in a B Cell-Intrinsic Manner. J Immunol 2010, 1-6.

12. Tortola $\mathrm{L}$, et al: $\mathrm{IL}-21$ induces death of marginal zone $B$ cells during chronic inflammation. Blood 2010, 116:5200-5207.

\section{0}

New insights into the role and signalling processes of gp130

Stefan Rose-John

Department of Biochemistry, Christian-Albrechts-Universität, 24098 Kiel, Germany

Arthritis Research \& Therapy 2011, 13(Suppl 2):010

Cytokine receptors exist in membrane bound and soluble form. While most soluble receptors are antagonists, some soluble receptors are agonists like soluble receptors of the gp130 cytokine family. In vivo, the IL-6/soluble IL-6R complex stimulates several types of target cells not stimulated by IL- 6 alone, since they do not express the membrane bound IL-6R. This process has been named trans-signaling [1]

We have shown that soluble gp130 is the natural inhibitor of IL-6/soluble IL-6R complex responses. The recombinant soluble gp130 protein is a molecular tool to discriminate between gp130 responses via membrane bound and soluble IL-6R responses. We have constructed a fusion of soluble gp130 and the Fc portion of human IgG1. This sgp130Fc protein proved to be efficient in blocking responses via the IL-6/soluble IL-6R complex without affecting IL- 6 responses, which are mediated via the membrane bound IL-6R [1].

The soluble IL-6R is mostly generated by proteolysis of the IL-6R transmembrane protein. Shedding of the IL-6R is mediated by the metalloprotease ADAM17, which is also responsible for the cleavage of TNF $\alpha$ and ligands of the EGF-R. Consequently, activation of ADAM17 has different effects on the activation of the immune response as well as on induction of regenerative responses $[2,3]$.

Interestingly, depending on the animal model used, global blockade of IL-6 signaling by neutralizing monoclonal antibodies and selective blockade of
IL-6 trans-signaling can lead to different consequences. We could recently show that inhibition of IL-6 trans-signaling was beneficial in a caecum ligation puncture model whereas global IL- 6 blockade showed no benefit in survival of the animals [4]. In contrast, in a sepsis model induced by a bolus injection of LPS, both, global blockade of IL- 6 signaling by neutralizing monoclonal antibodies and selective blockade of IL-6 transsignaling proved effective in preventing the death of the animals [5]. Also various infection models suggest a different outcome of global blockade of IL-6 as compared to selective IL- 6 trans-signaling inhibition.

We could show that the extent of IL- 6 trans-signaling in chronic inflammatory diseases and cancer is controlled by the soluble IL-6R. Using the sgp $130 \mathrm{Fc}$ protein or sgp130Fc transgenic mice we demonstrate that in several chronic inflammatory diseases and cancers including inflammatory bowel disease, peritonitis, rheumatoid arthritis, colon cancer, ovarian cancer and pancreatic cancer, that IL-6 trans-signaling via the soluble IL-6R is a crucial step in the development and the progression of the disease. Therefore, sgp $130 \mathrm{Fc}$ is a novel therapeutic agent for the treatment of chronic inflammatory diseases and cancer [1,6-8].

\section{References}

1. Jones SA, Scheller J, Rose-John S: Therapeutic strategies for the clinical blockade of IL-6/gp130 signaling. J Clin Invest 2011 in press.

2. Chalaris A, Adam N, Sina C, Rosenstiel P, Lehmann J, Schirmacher $P$, Hartmann D, Cichy J, Gavrilova O, Schreiber S, Jostock T, Matthews V, Häsler R, Becker C, Neurath MF, Reiß K, Saftig P, Scheller J, Rose-John S: Critical Role of the Disintegrin Metalloprotease ADAM17 for Intestinal Inflammation and Regeneration in Mice. J Exp Med 2010, 207:1617-1624.

3. Scheller J, Chalaris A, Garbers C, Rose-John S: ADAM17: a molecular switch to control inflammation and tissue regeneration. Trends Immunol 2011 in press.

4. Barkhausen $T$, van Griensven M, Vonberg R-P, Dorsch M, Waetzig GH, Seegert D, Chalaris A, Scheller J, Rose-John S, Hildebrand F, Krettek C, Tschernig T: Selective blockade of IL-6 trans-signaling improves survival in a murine polymicrobial sepsis model. Crit Care Med 2011, 39:1407-1413.

5. Greenhill CJ, Rose-John S, Lissilaa R, Ferlin W, Ernst M, Hertzog PJ, Mansell A, Jenkins BJ: IL-6 trans-signaling modulates TLR4-dependent inflammatory responses via STAT3. J Immunol 2011, 186:1199-208.

6. Lesina M, Kurkowski MU, Ludes K, Rose-John S, Treiber M, Klöppel G, Yoshimura A, Reindl W, Sipos B, Akira S, Schmid RM, Algül H: Stat3/Socs3 activation by IL- 6 transsignaling promotes progression of pancreatic intraepithelial neoplasia and development of pancreatic cancer. Cancer Cell 2011, 19:456-469.

7. Schiechl G, Bauer B, Fuss IJ, Lang SA, Moser C, Rose-John S, Neurath MF, Geissler E, Schlitt HJ, Strober W, Fichtner-Feigl S: Tumor growth in murine ulcerative colitis depends on innate signaling of colonic F4/80+ CD11bhigh Gr1 low macrophages. J Clin Invest 2011, 121:1692-1708.

8. Lo C-W, Chen MW, Hsiao M, Wang S, Chen C-A, Hsiao S-M, Chang J-S, Lai T-C, Rose-John S, Kuo M-L, Wei L-H: Role of IL-6 Trans Signaling in Malignant Ascites Formation and Ovarian Cancer Progression. Cancer Res 2011, 71:424-34

\section{1}

Discovery of stat3 signalling and its clinical relevance

Friedemann Horn

Leipzig, Germany

Arthritis Research \& Therapy 2011, 13(Suppl 2):011

Abstract not submitted at time of publication

\section{2}

Cytokine imprinting - mechanisms for memory

Andreas Radbruch

Deutsches Rheuma-Forschungszentrum Berlin, ein Leibniz Institut,

Charitéplatz 1, 10117 Berlin, Germany

Arthritis Research \& Therapy 2011, 13(Suppl 2):012

Imprinting of T helper lymphocytes for the reexpression of cytokines is crucial for protection against recurring pathogens but also can be a driving force of chronic inflammation. Th1 and Th17 cells are distinct lineages of proinflammatory effector/memory cells, imprinted for reexpression of interferon- $\gamma$ (IFN- $\gamma$ ) and interleukin-17 (IL-17), by upregulated expression of the transcription factors T-bet and ROR $\gamma \mathrm{t}$, respectively. Imprinting here 
means that while expression of the cytokine genes upon primary instruction requires signals from both, the T cell receptor and receptors for instructing cytokines, reexpression requires only $\mathrm{T}$ cell receptor signaling in reactivated effector/memory T cells. Interleukin-12 (IL-12) and IFN- $\gamma$ are essential instruction signals for the differentiation of Th1 cells and the imprinting of the Ifng gene. In activated naïve T cells, IFN- $\gamma$ induces the central Th1 transcription factor T-bet, while T-bet induces the expression of IFN- $\gamma$, in a positive, $T$ cell receptor dependent feedback loop polarizing the $T$ cell into Th1 differentiation. At this time, expression of the IL-12 receptor $\beta 2$ chain (IL12R $\beta 2$ ) is repressed by T cell receptor signaling. After TCR signaling has ceased, i.e. the antigen is eliminated, the IL12RB2 chain is expressed and IL-12 triggers a second wave of T-bet expression. This "late" T-bet expression coincides with expression of the transcription factors $\mathrm{HIX}$ and Runx3, and it is required for imprinting of Th1 cells for the reexpression of IFN- $\gamma$. The signals required for Th17 differentiation and imprinting are less clearly defined. While signals such as TGF- $\beta$ and IL- 6 lead to the differentiation of IL-17 expressing cell in vitro, such cells fail to reexpress IL-17 in the absence of these instructive signals. In contrast, in vivo generated Th17 cells, have a stable memory for reexpression of IL-17 in vitro, upon restimulation by antigen. Such cells are refractory to Th1 or Th2 polarizing signals. Th cells coexpressing IFN- $\gamma$ and IL-17 have been observed in vivo. Ex vivo isolated Th17 cells can be converted into $\mathrm{Th} 1+17$ cells by combined IFN- $\gamma$ and IL- 12 signaling. IFN- $\gamma$ is required to upregulate expression of the IL12R $\beta 2$ chain, and IL-12 for Th1 differentiation. These Th1+17 cells stably coexpress RORyt and T-bet on the single cell level, and are imprinted for reexpression of both IFN- $\gamma$ and IL-17. Thus, for T lymphocytes, polarization and imprinting of inflammatory responses is regulated by dynamic interaction of IFN- $\gamma$ and IL-12, and regulation of expression of the IL-12 receptor.

\section{3}

Cytokine induced molecular mechanisms of bone/cartilage metabolism Georg Schett

Erlangen, Germany

Arthritis Research \& Therapy 2011, 13(Suppl 2):013

Abstract not submitted at time of publication

\section{4}

Immunomodulatory cytokines: directing and controlling immune activation

Fillatreau Simon

Deutsche Rheuma-Forschungszentrum, a Leibniz institute. Chariteplatz 1,

10117 Berlin, Germany

E-mail: fillatreau@drfz.de

Arthritis Research \& Therapy 2011, 13(Suppl 2):014

A hallmark of immunity is the production of a multi-faceted array of inflammatory cytokines that exerts decisive influence on innate and adaptive immune responses. The importance of these mediators of intercellular communication in autoimmunity is illustrated by the beneficial effects resulting from blockade of single cytokines such as TNF or IL-6 in these diseases. $B$ lymphocytes can also play pathogenic roles during autoimmune disease because $B$ cell depletion often led to amelioration of disease in patients treated with rituximab [1]. The pathogenic functions of $B$ cells during autoimmune diseases are poorly understood. They might involve autoantibody production, yet the beneficial effects resulting from the depletion of $B$ cells usually preceded reduction in autoantibody titers [2]. We recently demonstrated that the pathogenic activities of B cells during experimental autoimmune encephalomyelitis (EAE), the primary animal model for multiple sclerosis (MS), were largely mediated through provision of inflammatory cytokines. B cells from MS patients also produced enhanced amounts of inflammatory cytokines, compared to $B$ cells from healthy individuals, and this abnormality was corrected through $B$ cell depletion i.e. $B$ cells returning at 1 year after rituximab treatment showed a normalized cytokine response. Inflammatory processes are usually balanced by counter-regulatory circuits involving anti-inflammatory cytokines such as interleukin (IL)-10 [3,4]. We previously demonstrated that IL-10 production by B lymphocytes played a determinant role for the resolution of EAE [5]. Accordingly, IL-10 might provide a powerful means for controlling pathogenic immune reactions. However, administration of IL-10 into patients achieved little beneficial effects in the clinic, asking for a better understanding of the immunosuppressive biology of this molecule. To this end, we pursued the characterization of IL-10-producing B cells in a model of infection by the intracellular bacterial pathogen Salmonella typhimurium. Using a novel strain of IL-10.eGFP knock-in mice to facilitate the tracking of these cells, we could identify IL-10 producing B cells already within 24 hours after infection in spleen, and demonstrated that all IL-10+ $\mathrm{B}$ cells expressed the cell surface receptor CD138, which is a distinctive marker of antibody secreting cells [6,7]. IL-10 expression was undetectable in other cell types such as dendritic cells, macrophages, or $\mathrm{T}$ cells at this stage, implying that plasmablasts were the first producers of IL-10 during immune reactions. These data suggest that IL-10 might be needed at a very early stage of immune reactions to be suppressive. Collectively, our data showed that B cells have a dual role during autoimmune diseases, acting both as drivers and regulators of pathogenesis, and identified cytokine production as core mechanisms in these complex functions.

References

1. Hoehlig K, Lampropoulou V, Roch T, Neves P, Calderon-Gomez E, Anderton SM, Steinhoff U, Fillatreau S: Immune regulation by B cells and antibodies a view towards the clinic. Adv Immunol 2008, 98:1-38.

2. Martin F, Chan AC: B cell immunobiology in disease: evolving concepts from the clinic. Annu Rev Immunol 2006, 24:467-496.

3. Lampropoulou V, Calderon-Gomez E, Roch T, Neves P, Shen P, Stervbo U, Boudinot $P$, Anderton SM, Fillatreau S: Suppressive functions of activated $B$ cells in autoimmune diseases reveal the dual roles of Toll-like receptors in immunity. Immunol Rev 233:146-161.

4. Anderton SM, Fillatreau S: Activated B cells in autoimmune diseases: the case for a regulatory role. Nat Clin Pract Rheumatol 2008, 4:657-666.

5. Fillatreau S, Sweenie CH, McGeachy MJ, Gray D, Anderton SM: B cells regulate autoimmunity by provision of IL-10. Nat Immunol 2002 3:944-950

6. Neves P, Lampropoulou V, Calderon-Gomez E, Roch T, Stervbo U, Shen P, Kuhl AA, Loddenkemper C, Haury M, Nedospasov SA, et al: Signaling via the MyD88 adaptor protein in B cells suppresses protective immunity during Salmonella typhimurium infection. Immunity 33:777-790.

7. Fillatreau S: Novel regulatory functions for Toll-like receptor-activated B cells during intracellular bacterial infection. Immunol Rev 240:52-71.

\section{5}

Interleukin-37 as fundamental inhibitor of innate immunity

P Bufler

Dr. von Hauner Children's Hospital, Ludwig-Maximilians-University, Munich, Germany Arthritis Research \& Therapy 2011, 13(Suppl 2):015

The interleukin-1 (IL-1) family of ligands has 11 members of which most are proinflammatory. The receptors, signaling pathways, and functions of the classical family members (IL-1 $\alpha, \mathrm{IL}-1 \beta$ and IL-18) have been studied extensively. However, knowledge of Interleukin-37 (IL-37/IL-1F7), which was first identified by in silico research in 2000 remains limited.

IL-37 shares critical amino acid residues with IL-18 and binds to the IL-18binding protein enhancing its ability to inhibit IL-18-induced interferon- $\gamma$. Data suggest that IL-37 also binds to the IL-18R $\alpha$ and, for its antiinflammatory properties, likely recruits an accessory receptor chain with inhibitory properties, such as the single Ig IL-1 related receptor. We recently reported that overexpression of IL-37 in cells of monocytic or epithelial origin almost completely abolishes the production of proinflammatory cytokines as IL-1 $\alpha / \beta$, TNF $\alpha$, IL- 6 and IL- 8 in response to TLR-ligands or IL- $1 \beta$. Anti-inflammatory cytokines were unaffected. Vice versa, functional knockdown of IL-37 in primary human cells by siRNA increased the production of proinflammatory cytokines. IL-37tg mice are protected against LPS-induced shock. Thus IL-37 is a fundamental inhibitor of innate immune responses.

IL-37 protein is expressed in human monocytes and upregulated by LPS Similarly to IL- $1 \alpha$ and IL-33, IL-37 is expressed intracellularly and translocates to the nucleus upon cell stimulation in a caspase-1 dependent manner. IL-37 interacts inside the cell with Smad3 and inflammation in IL-37tg mice is increased when endogenous Smad3 is depleted. IL-37 is also secreted in the supernatant of stimulated 
transfected cells or peripheral mononuclear blood cells. However, the extracellular functionality of IL-37 is still elusive.

\section{6}

B cell directed cytokines

Peter E Lipsky

Charlottesville, VA, USA

Arthritis Research \& Therapy 2011, 13(Suppl 2):016

The prototypic autoimmune disease, systemic lupus erythematosus (SLE), is known to be associated with polyclonal B cell activation [1]. A number of cytokines play essential roles in driving or supporting $B$ cell responses, and are, therefore, candidate targets for controlling the B cell activity in SLE. Among these cytokines are IL-6, IL-21 and BAFF/BLyS. IL- 6 is a pleiotropic cytokine with effects on a number of cell types, including $B$ cells, where it plays as essential role in plasma cell differentiation and survival. Blocking IL-6 activity with tocilizumab is approved for treatment of rheumatoid arthritis and preliminary data suggest that it might also be effective in the treatment of SLE [2]. Importantly, treatment of SLE is associated with a decline in anti-DNA antibodies and also a decrease in the frequency of circulating plasma cells, suggesting that at least part of its action relates to an impact on terminal differentiation of B cells into plasma cells. IL-21 is a type I cytokine with effects on a number of cell types, including a nonredundant requirement in $\mathrm{B}$ cell activation and differentiation into plasma cells [3]. Levels of IL-21 are elevated in a number of animal models of lupus and also in human SLE. Blocking IL-21 is effective in animal models of lupus, whereas polymorphisms in both the IL-21 gene and in the IL-21 receptor gene are associated with human SLE. Trials of blocking IL-21 in human SLE have not yet begun. BAFF/BLyS(TNFSF13b) is a TNF family member that binds to 3 separate receptors and contributes to both naïve $B$ cell and plasma cell survival [4]. Overexpression of BAFF/BLyS in mice leads to a lupus-like disorder, whereas blocking this cytokine ameliorates lupus in mouse models. Clinical trials in human SLE of a blocking monoclonal antibody, belimumab, have shown moderate clinical benefit associated with decreases in anti-DNA antibody titers and a decrease in circulating naïve $B$ cells and plasma cells. These results led to the approval of this product for the treatment of SLE in the US. The approval of belimumab for treatment of SLE has confirmed that targeting B cells can be effective in treating this disease and has provided impetus for the development of additional B cell-directed therapies aimed at blocking cytokines involved in B cell survival and/or functional responsiveness.

\section{References}

1. Dorner T, et al: Arthritis Res Ther 2009, 11:247-257.

2. Illei GG, et al: Arthritis Rheum 2010, 62:542-552.

3. Ettinger R, et al: Ann Rheum Dis 2008, 67:83-86

4. Cancro MP, et al: J Clin Invest 2009, 119:1066-1073.

Cite abstracts in this supplement using the relevant abstract number, e.g.: Lipsky: B cell directed cytokines. Arthritis Research \& Therapy 2011, 13 (Suppl 2):016 